\title{
Morfometria de ovários de fêmeas zebu Bos taurus indicus coletados em matadouro
}

\author{
Morphometry of ovaries of female zebu Bos taurus indicus collected in slaughterhouse \\ Marcelo George Mungai Chacur', Nayara Coutinho Valentim², Ana Inês \\ Sanchez Martinez ${ }^{3}$, Raimundo Alberto Tostes ${ }^{4}$ \& Sérgio do Nascimento Kronka ${ }^{5}$
}

\begin{abstract}
RESUMO
O estudo da morfometria ovariana está diretamente ligado as suas aplicações práticas para realizar e interpretar os achados dos exames ginecológicos em vacas. O objetivo do trabalho foi estudar as características morfométricas de ovários de vacas zebu não prenhes, criadas na microrregião de Presidente Prudente-SP. Foram coletados 114 pares de ovários em abatedouro, mensurados quanto a sua espessura, comprimento, largura e volume; diâmetro e volume do folículo, e diâmetro e área do corpo lúteo. Foi observada diferença significativa para largura $(1,95 \mathrm{~cm}$ e $1,83 \mathrm{~cm})$ e volume $(7,26 \mathrm{~mL}$ e $6,23 \mathrm{~mL})$ dos ovários esquerdo e direito, respectivamente. Para o tamanho e volume dos folículos; e diâmetro e área dos corpos lúteos, não houve diferença significativa entre os lados. Houve correlação positiva $(\mathrm{p}<0,01)$ entre o volume do ovário esquerdo e a área do corpo lúteo. Na presença de folículos com diâmetro igual ou superior a $9 \mathrm{~mm}$, o corpo lúteo do tipo maciço e protruso presente em 43,39\% dos 53 ovários, predominou em relação ao tipo cavitário e incluso. Dos 84 ovários com corpos lúteos 26,20\% eram do tipo incluso. Conclui-se que a presença de corpos lúteos inclusos, nas vacas zebu, pode resultar em falha durante o exame de palpação retal para estimar a atividade ovariana.
\end{abstract}

Descritores: morfometria ovariana, Bos taurus indicus, folículo, corpo lúteo.

\section{ABSTRACT}

The study of ovarian morphometry is directly related to its practical applications to interpret the examination of the reproductive tract of the cow. The objective of this work was to study the morphometric characteristics of ovaries of not pregnant zebu cows raised in the microregion of Presidente Prudente-SP. One hundred fourteen pairs of ovaries were collected from slaughterhouse, measured in thickness, length, width and volume, diameter and volume of the follicle, diameter and area of the corpora luteum. Significative differences were observed for widths of $(1,95 \mathrm{~cm}$ and 1,83 cm) and volume of $(7,26 \mathrm{~mL}$ and 6,23 $\mathrm{mL})$ of the ovaries left and right, respectively. For the size and volume of the follicles, and diameter and area of the corpora luteum, there was no significative difference between the sides. There was correlation $(p<0,01)$ between the volume of the left ovary and the area of the corpora luteum, in the presence of follicles with diameters equal or greater than $9 \mathrm{~mm}$, the corpora luteum of the massive and protuberant type were present in $43,39 \%$ of 53 ovaries, predominated in relation to type of cavity and inclusion. In conclusion, the presence of the included corpora luteum in zebu cows, could result in a fault during the rectal palpation exam to estimate the ovarian activity.

Key words: ovarian morphometry, Bos taurus indicus, follicle, corpora luteum. 


\section{INTRODUÇÃO}

Os ovários são órgãos pares do sistema reprodutor feminino, com localização e tamanho variando entre as espécies [13]. Nos bovinos, as gônadas tem em média 3,0 a $4,5 \mathrm{~cm}$ de comprimento, 1,5 a $2,0 \mathrm{~cm}$ de largura e 2,0 a 2,8 de espessura [19], com coloração rósea clara devido à albugínea [8]. Possui funções de produzir hormônios e liberar óvulos, histologicamente se divide em medular e cortical, circundado pelo epitélio germinativo com folículos e corpos lúteos [6].

$\mathrm{Na}$ formação do corpo lúteo (CL), o tecido pode proliferar ocupando apenas a área interior do ovário, o que resulta em CL incluso ou extrapolando para fora do ovário formando um ápice na superfície, classificado como CL protruso [14]. A identificação do CL em zebus geralmente é realizada por meio de ultra-som, devido à pequena precisão em identificar e caracterizar a fase progesterônica, quando da utilização da palpação retal em exames ginecológicos, em vacas com CL de pequeno diâmetro ou inclusos [16].

As mudanças cíclicas que ocorrem no ovário em função da formação e regressão de corpos lúteos e do surgimento das ondas de crescimento folicular podem dificultar a interpretação dos achados clínicos no exame ginecológico [13].

A presença de cavidade pode ser observada no centro de alguns corpos lúteos, em bovinos após o abate, tendo maior incidência no CL em desenvolvimento, e menor no já desenvolvido [15].

O objetivo do trabalho foi estudar as características morfométricas de ovários de vacas zebu não prenhes, criadas na microrregião de Presidente Prudente-SP.

\section{MATERIAIS E MÉTODOS}

Foi realizado um estudo por meio de coleta de 114 pares de ovários, de vacas zebus não prenhes, recuperados em matadouro na cidade de Presidente Prudente, São Paulo, no período de agosto a novembro de 2004. As 114 fêmeas apresentavam escore corporal homogêneo, com média de 3,5 na escala de 1 a 5 [17]. Os ovários foram coletados imediatamente após o abate e evisceração dos animais, identificados quanto ao lado em ovário direito (OD) e ovário esquerdo (OE); armazenados e transportados em solução salina ${ }^{1}$ a $37^{\circ} \mathrm{C}$ para os laboratórios de Reprodução Animal e de Patologia Veterinária da UNOESTE.

Todos os ovários foram mensurados com auxílio de um paquímetro ${ }^{2}$, verificando-se o comprimento $(\mathrm{cm})$ no eixo maior, largura $(\mathrm{cm})$ no eixo entre o pedículo do ovário e a extremidade oposta; e espessura (cm) no eixo de $90^{\circ} \mathrm{em}$ relação ao eixo da largura. $\mathrm{O}$ volume $(\mathrm{mL})$ dos ovários foi obtido por meio da imersão individual dos mesmos em proveta ${ }^{3}$ graduada, contendo solução fisiológica.

O maior folículo presente nos ovários direito e esquerdo foi identificado e mensurado quanto ao seu diâmetro $(\mathrm{mm})$ e volume $(\mathrm{mL})$. A área de tecido luteal foi calculada a partir da área da elipse, definida pelo maior e menor diâmetros do corpo lúteo (CL), o qual foi classificado em CL incluso quando da ocupação total do tecido luteal abaixo da superfície do ovário ou CL protruso apresentando uma porção luteal acima da superfície do ovário, segundo $[14,16]$. Os corpos lúteos foram classificados em CL cavitário, quando da presença de cavidade central ou de CL maciço, onde a totalidade da massa luteal se mostrou compacta.

Os resultados das médias foram submetidos à análise de variância pelo teste $\mathrm{F}$, de acordo com um delineamento inteiramente casualizado [2], cujo modelo matemático é:

$$
\begin{aligned}
& \text { xij }=m+t i+\text { eij, em que: } \\
& \text { xij = valor observado na parcela do lado i e repetição } \\
& j ; m=\text { média geral; ti = efeito do lado } i ; \text { eij }=\text { efeito do } \\
& \text { acaso. }
\end{aligned}
$$

Para alguns pares de medições de interesse foi calculado o coeficiente de correlação linear através do teste $t$ de Student. Para o número de corpos lúteos foi aplicado o teste de qui-quadrado $\left(\chi^{2}\right)$.

\section{RESULTADOS}

Para a largura e o volume dos ovários, houve diferença $(p<0,05)$ entre os lados esquerdo e direito com $1,95 \mathrm{~cm}$ e $1,83 \mathrm{~cm}$; e de $7,26 \mathrm{~mL}$ e $6,23 \mathrm{~mL}$, respectivamente. Já para a espessura e o comprimento não houve diferença $(p>0,05)$ entre os lados. $O$ volume ovariano revelou um coeficiente de variação $(\mathrm{CV})$ de $54,10 \%$, superior aos coeficientes para a largura, espessura e comprimento dos ovários. Com relação ao tamanho e volume dos folículos, e diâmetro e área dos corpos lúteos, não houve diferença significativa entre os lados direito e esquerdo. Os maiores coeficientes de variação foram encontrados para o volume folicular $(\mathrm{CV}=143,14 \%)$ e área do CL (CV=49,33\%) (Tabela 1$)$.

Para as correlações entre as estruturas encontradas nos ovários, dentre elas volume ovariano (VO) 
Tabela 1. Médias e resultados da análise de variância do volume, comprimento, largura e altura do ovário; tamanho e volume folicular; diâmetro e área do corpo lúteo de 114 pares de ovários de vacas zebus não prenhes, recuperados em matadouro, Presidente Prudente-SP (2004).

\begin{tabular}{|c|c|c|c|c|c|c|c|c|}
\hline \multirow[b]{2}{*}{ Tratamentos } & \multicolumn{4}{|c|}{ Ovário } & \multicolumn{2}{|c|}{ Folículo } & \multicolumn{2}{|c|}{ Corpo lúteo } \\
\hline & $\begin{array}{c}\text { Volume } \\
(\mathrm{mL})\end{array}$ & $\begin{array}{l}\text { Largura } \\
(\mathrm{cm})\end{array}$ & $\begin{array}{l}\text { Espessura } \\
\text { (cm) }\end{array}$ & $\begin{array}{l}\text { Comprimento } \\
\text { (cm) }\end{array}$ & $\begin{array}{l}\text { Tamanho } \\
(\mathrm{mm})\end{array}$ & $\begin{array}{c}\text { Volume } \\
\left(\mathrm{cm}^{3}\right)\end{array}$ & $\begin{array}{l}\text { Diâmetro } \\
\text { (mm) }\end{array}$ & $\begin{array}{l}\text { Área } \\
\left(\mathrm{cm}^{2}\right)\end{array}$ \\
\hline Lado esquerdo & $7,26^{a}$ & $1,95^{\mathrm{a}}$ & $1,65^{a}$ & $2,75^{a}$ & $8,09^{a}$ & $0,49^{a}$ & $16,70^{\mathrm{a}}$ & $2,32^{a}$ \\
\hline Lado direito & $6,23^{b}$ & $1,83^{b}$ & $1,56^{a}$ & $2,80^{a}$ & $8,36^{a}$ & $0,53^{a}$ & $15,75^{\mathrm{a}}$ & $2,14^{a}$ \\
\hline Teste F & $6,23^{*}$ & $4,21^{*}$ & $2,50^{\mathrm{NS}}$ & $0,41^{\mathrm{NS}}$ & $0,22^{\mathrm{NS}}$ & $0,21^{\mathrm{NS}}$ & $0,80^{\mathrm{NS}}$ & $0,54^{\mathrm{NS}}$ \\
\hline Média geral & 6,74 & 1,89 & 1,61 & 2,78 & 8,22 & 0,51 & 16,22 & 2,23 \\
\hline Desvio padrão & 3,04 & 0,45 & 0,38 & 0,61 & 3,99 & 0,73 & 4,63 & 1,10 \\
\hline C.V. (\%) & 54,10 & 23,81 & 23,60 & 21,94 & 48,54 & 143,14 & 28,55 & 49,33 \\
\hline
\end{tabular}

e folicular; VO e área de CL; e tamanho folicular e diâmetro de CL não houve diferença $(\mathrm{p}>0,05)$. Por outro lado, houve correlação positiva $(\mathrm{p}<0,01)$ entre o volume do ovário esquerdo e a área do CL (Tabela 2).

Na presença de folículos com diâmetro igual ou superior a $9 \mathrm{~mm}$, encontrados em $53(23,24 \%)$ dos 228 ovários avaliados, não houve diferença $(\mathrm{p}>0,05)$ para o número de corpos lúteos cavitários inclusos ou protrusos; e maciços inclusos ou protrusos, entre os ovários direito e esquerdo. O CL do tipo maciço e protruso predominou sobre os outros tipos, estando presente em $23(43,39 \%)$ dos 53 ovários. Por outro lado, $19(35,84 \%)$ dos 53 ovários, revelaram a presença de corpos lúteos cavitários ou maciços e inclusos (Tabela 3).

Tabela 2. Correlações entre o volume do ovário direito (OD) e esquerdo (OE) e volume do folículo direito (FOLD) e esquerdo (FOLE); área e diâmetro do corpo lúteo (CL), de 114 pares de ovários de vacas zebus não prenhes, recuperados em matadouro, Presidente Prudente-SP (2004).

\begin{tabular}{cc}
\hline Parâmetros & Correlações \\
\hline Volume OE e volume FOL & $0,14^{\mathrm{NS}}$ \\
Volume OD e volume FOL & $0,08^{\mathrm{NS}}$ \\
Volume OE e área CL & $0,46^{* *}$ \\
Volume OD e área CL & $0,04^{\mathrm{NS}}$ \\
Tamanho FOLD e diâmetro CL & $0,21^{\mathrm{NS}}$ \\
Tamanho FOLE e diâmetro CL & $0,04^{\mathrm{NS}}$ \\
Volume FOLE e área CL & $-0,16^{\mathrm{NS}}$ \\
Volume FOLD e área CL & $0,22^{\mathrm{NS}}$ \\
\hline **ignificativo a $1 \%$ N não significativo a $5 \%$. &
\end{tabular}

Para o número e porcentagem de corpos lúteos, presentes em 84 ovários de um total de 228 , ou seja $36,84 \%$, observaram-se $55(65,47 \%)$ no lado esquerdo e 29 (34,53\%) no lado direito. Dos 84 ovários com CL, $22(26,20 \%)$ eram do tipo incluso e $62(73,80 \%)$ do tipo protruso (Tabela 4).

\section{DISCUSSÃO}

A despeito de vários estudos clínicos e epidemiológicos sobre as estruturas ovarianas em vacas, há a necessidade de uma caracterização da realidade regional, sendo importante ressaltar o rebanho do Oeste do Estado de São Paulo na pecuária nacional. O plantel bovino paulista soma aproximadamente 13 milhões de cabeças, cerca de 6\% lotadas na microrregião de Presidente Prudente-SP, exibindo a maior densidade de

Tabela 3. Número de corpos lúteos (CL) cavitários ou maciços; e inclusos ou protrusos de 114 pares de ovários de vacas zebus não prenhes, recuperados em matadouro, na presença de folículos com diâmetros iguais ou superiores a 9 mm, Presidente Prudente-SP (2004).

\begin{tabular}{lcccc}
\hline & $\begin{array}{c}\text { Ovário } \\
\text { Direito }\end{array}$ & $\begin{array}{c}\text { Ovário } \\
\text { Esquerdo }\end{array}$ & Total & $\begin{array}{l}\text { Teste de Qui- } \\
\text { quadrado }\left(\chi^{2}\right)\end{array}$ \\
\hline $\begin{array}{l}\text { CL cavitário } \\
\text { e incluso }\end{array}$ & 0 & 3 & 3 & $\chi^{2}=3,00^{\mathrm{NS}}$ \\
$\begin{array}{l}\text { CL cavitário } \\
\text { e protruso }\end{array}$ & 3 & 8 & 11 & $\chi^{2}=2,27^{\mathrm{NS}}$ \\
$\begin{array}{l}\text { CL maciço } \\
\text { e incluso }\end{array}$ & 8 & 8 & 16 & $\chi^{2}=0,00^{\mathrm{NS}}$ \\
$\begin{array}{l}\text { CL maciço } \\
\text { e protruso }\end{array}$ & 10 & 13 & 23 & $\chi^{2 e}=0,39^{\mathrm{NS}}$ \\
\hline \begin{tabular}{l} 
Total \\
\hline Nsnão significativo a $5 \%$.
\end{tabular} & & 53 & \\
\hline
\end{tabular}


Tabela 4. Número e porcentagem de corpos lúteos (CL) maciços ou cavitários; inclusos ou protrusos no ovário direito (OD) ou esquerdo (OE) de 84 ovários de vacas zebus não prenhes, recuperados em matadouro, Presidente Prudente - SP (2004).

\begin{tabular}{lcc}
\hline Característica do Ovário & $\mathbf{n}$ & (\%) \\
\hline CLOE & 55 & 65,47 \\
CLOD & 29 & 34,53 \\
\hline Total de ovários com CL & 84 & 100 \\
\hline CL cavitário & 15 & 17,85 \\
CL maciço & 69 & 82,14 \\
CL incluso & 22 & 26,19 \\
CL protruso & 62 & 73,80 \\
\hline Total de ovários com CL & 84 & \\
\hline
\end{tabular}

cabeças no estado [7]. Dentro dessa realidade é necessário considerar que a caracterização morfométrica dos ovários bem como das suas estruturas serve como subsídio para interpretar os achados clínicos durante o exame ginecológico.

No presente estudo, a média da largura ovariana foi maior e as do comprimento e espessura menores, quando comparados com ovários de vacas azebuadas, recuperados em matadouro, obtendo valores de 1,$49 ; 2,81$ e $1,72 \mathrm{~cm}$, respectivamente, para o ovário esquerdo; e 1,57; 3,01 e 1,85 cm para o direito [11].

Medidas inferiores para espessura e comprimento dos ovários também foram observadas na Tabela 1, frente aos valores de 2,0 a $2,8 \mathrm{~cm}$ de espessura e 3,0 a $4,5 \mathrm{~cm}$ de comprimento para vacas de origem européia Bos taurus taurus [19].

Em contrapartida, as médias descritas na Tabela 1 para o comprimento e espessura dos ovários entre os lados direito e esquerdo não apresentaram diferença $(p>0,05)$. Concordando com os resultados obtidos para vacas zebus, com medidas de 2,57 $\pm 0,70 \mathrm{~cm}$ de comprimento para o ovário esquerdo e $2,62 \pm 0,54 \mathrm{~cm}$ para o direito; e valores médios de 1,22 $\pm 0,39 \mathrm{~cm}$ para a espessura do ovário esquerdo e 1,21 $\pm 0,31 \mathrm{~cm}$ para o direito [14].

Por outro lado o presente trabalho revelou comprimentos ovarianos superiores (Tabela 1) em relação aos $2,01 \pm 0,74 \mathrm{~cm}$ e $2,42 \pm 0,89 \mathrm{~cm}$ para as gônadas esquerda e direita, respectivamente para vacas $3 / 8$ zebu x $5 / 8$ taurino [20].
Sugere-se que as medidas do presente estudo para o comprimento e espessura dos ovários possam estar relacionadas com a origem genética das vacas zebuínas, uma vez que os autores supracitados descreveram medidas ovarianas de vacas mestiças Bos taurus taurus $x$ Bos taurus indicus denominadas de azebuadas, e de fêmeas Bos taurus taurus, às quais de modo geral possuem ovários com maiores dimensões. Quando essas dimensões foram comparadas com as obtidas de animais de origem zebuína, verificou-se similaridade.

Houve diferença $(\mathrm{p}<0,05)$ entre os lados direito e esquerdo para o volume e largura dos ovários (Tabela 1). Em contrapartida, não houve diferença $(p>0,05)$ entre os lados, com $6,37 \pm 2,83 \mathrm{~mL}$ para o esquerdo; e 7,16 $\pm 3,49 \mathrm{~mL}$ no direito; o mesmo ocorrendo com a largura, sendo de $1,61 \pm 0,32 \mathrm{~cm}$ e $1,71 \pm 0,36 \mathrm{~cm}$ para os ovários esquerdo e direito, respectivamente [14]. Diferença essa associada aos 65,47\% de corpos lúteos presentes no lado esquerdo, sendo $73,80 \%$ deles protrusos, levando ao aumento significativo das dimensões ovarianas (Tabela 4).

O corpo lúteo funciona como um órgão endócrino transitório com um papel essencial na função reprodutiva [4]. Basicamente a estrutura do CL consta de células da teca e da granulosa, sendo que as células da granulosa iniciam a luteinização na onda préovulatória de LH e é incrementada com a ovulação [3].

Não houve diferença entre os lados direito e esquerdo $(p>0,05)$ para o diâmetro do corpo lúteo, o que corrobora os achados em vacas zebu não prenhes, cujas médias foram de $1,64 \pm 0,30 \mathrm{~cm}$ e $1,53 \pm 0,34 \mathrm{~cm}$ para os lados direito e esquerdo, respectivamente.

A média do tamanho dos maiores folículos $8,22 \pm 3,99 \mathrm{~mm}$ influenciou de forma negativa no volume do ovário direito $6,23 \mathrm{~mL}$. Essa observação pode parecer contraditória, uma vez que a presença de um folículo de maior tamanho deveria resultar em um ovário com dimensões superiores. $\mathrm{O}$ folículo dominante determina a atresia dos folículos subordinados e inibe o recrutamento de novos folículos [1,5].

A mensuração do maior folículo ovariano encontrado, não revelou diferença $(p>0,05)$ entre os dois lados, o que diverge dos valores para ovários também recuperados em matadouro, sendo lado direito $9,06 \pm 0,69 \mathrm{~mm}$ e esquerdo $6,40 \pm 0,68 \mathrm{~mm}$, em vacas com atividade ovariana luteal cíclica [13].

No presente estudo valores intermediários para o tamanho do maior folículo são apresentados na Ta- 
bela 1 , em relação à maior e menor médias descritas [13]. Conforme ilustra a Tabela 2, houve correlação $(r=0,46 ; p<0,01)$ entre o volume do ovário esquerdo e área do corpo lúteo, concordando com o valor de $(\mathrm{r}=0,69 ; \mathrm{p}<0,01)$ [13].

Existem diferenças funcionais entre os corpos lúteos maciços e cavitários, mas a presença da cavidade não está relacionada com condições patológicas [15]. Corpos lúteos que apresentam cavidade podem ser encontrados em animais gestantes (86\%) e não gestantes (77\%), sendo sua provável origem atribuída à ocupação incompleta da cavidade folicular pelas células durante a luteinização [9]. Esses corpos lúteos foram classificados como cavitários quando o diâmetro de suas cavitações eram superiores a $1 \mathrm{~cm}$ [10]. O tamanho da cavidade está relacionado com o estágio de desenvolvimento do tecido luteal, sendo a mesma maior na fase de formação do CL em relação ao CL desenvolvido [15].

Os ovários com CL cavitário estiveram presentes em 26,41\% em relação ao total de corpos lúteos presentes (Tabela 3), similar aos relatos onde houve a predominância de corpos lúteos maciços $(58,7 \%)$ [15] e de $83,7 \%$ de CL maciços e de $16,3 \%$ de corpos lúteos cavitários em vacas zebu [14].

No presente estudo não houve diferença significativa entre os lados direito e esquerdo na presença de folículos com diâmetros iguais ou superiores a $9 \mathrm{~mm}$, nos ovários com corpos lúteos cavitários ou maciços (Tabela 3). Folículos são identificados no transcorrer do ciclo estral, vindo a sofrer atresia devido à produção de progesterona pelo CL, concordando com as observações para ovários obtidos de vacas da raça Holstein Friesian, recuperados em matadouro [15].
Conforme ilustrado na Tabela 4, houve maior número de corpos lúteos no ovário esquerdo $(65,47 \%)$ em relação ao ovário direito $(34,53 \%)$, o que difere de outros resultados que apontaram um predomínio de corpos lúteos no ovário direito $(65,10 \%)$ em relação ao ovário esquerdo $(34,90 \%)$ [12].

O presente trabalho revelou que $26,20 \%$ do total dos corpos lúteos eram do tipo incluso (Tabela 4) sendo esse um achado de utilização prática, uma vez que a estrutura luteinizada pode não ser detectada por meio da palpação retal, durante a avaliação ginecológica, podendo levar ao diagnóstico equivocado quanto à atividade ovariana luteal cíclica, concordando com outros estudos [17,21].

Devido ao avanço no conhecimento da morfofisiologia do ovário e seu emprego prático nas biotécnicas da reprodução das fêmeas, novos estudos devem ser realizados nesse sentido.

\section{CONCLUSÕES}

As características morfológicas do ovário variam em função da fase do ciclo estral, podendo ser utilizadas como parâmetro na avaliação clínica e funcional do órgão. A presença de corpos lúteos do tipo incluso nas vacas zebu sugere que a palpação retal das estruturas ovarianas nem sempre é eficaz para predizer a atividade ovariana luteal cíclica.

Agradecimentos. Ao Frigorífico Bon-Mart de Presidente Prudente-SP pelo apoio para a realização desse trabalho.

\section{NOTAS INFORMATIVAS}

${ }^{1}$ Prisfar, São Paulo/SP.

${ }^{2}$ Super Caliper, Mitutoyo, Japão.

${ }^{3}$ Pyrex, São Paulo/SP.

\section{REFERÊNCIAS}

1 Adams G.P., Kot K., Smith C.A. \& Ginther O.J. 1993. Selection of a dominant follicle and suppression of follicular growth in heifers. Animal Reproduction Science. 30: 259-271.

2 Banzatto D.A. \& Kronka S.N. 2006. Experimentação agrícola. 4.ed. Jaboticabal: FUNEP, 237p.

3 Davidson A.P. \& Stabenfeldt G.H. 1999. Controle da ovulação e do corpo lúteo. In: Tratado de fisiologia veterinária. 2.ed. Rio de Janeiro: Guanabara Koogan, pp.361-367.

4 Foley G.L. 1996. Pathology of the corpus luteum of cows. Theriogenology. 45: 1413-1428.

5 Ginther O.J. 1997. Emergency and deviation of follicles during the development of follicular waves in cattle. Theriogenology. 48: 75-87.

6 Hafez E.S.E. 2004. Reprodução Animal. 6.ed. São Paulo: Manole, 315p.

7 Instituto Brasileiro de Geografia e Estatística (IBGE) 1999. Pesquisa Pecuária Municipal. Disponível em:<http:// www.ibge.gov.br>. Acessado em 11/2005.

8 Junqueira L.C. \& Carneiro J.C. 1995. Histologia Básica. 8.ed. Rio de Janeiro: Guanabara Koogan, 433p. 
9 Kastelic J.P., Pierson R.A. \& Ginther O.J. 1990. Ultrasonic morphology of corpora lutea and central cavities during the estrour cycle and early pregnancy in heifers. Theriogenology. 33:487-498.

10 McEntee K. 1990. Reproductive Pathology of Domestic Animals. San Diego: Academic Press, 425p.

11 Megale F. \& Couto E.S. 1959. Aspectos anatômicos do aparelho reprodutor de vacas azebuadas abatidas em abatedouro. Arquivos da Escola Superior de Veterinária de Minas Gerais. 12: 529-535.

12 Mello V.F. 2003. Influência da receptora e do embrião sobre a viabilidade embrionária e sexo determinados através da ultrassonografia. 104f. São Paulo, SP. Dissertação (Mestrado em Medicina Veterinária) - Faculdade de Medicina Veterinária e Zootecnia, Universidade de São Paulo.

13 Nascimento A.P., Pinheiro N.L., Sales A. \& Viana J.H. 2003. Correlação morfométrica do ovário de fêmeas bovinas em diferentes estádios reprodutivos. Brazilian Journal of Veterinary Research and Animal Science. 40: 126-132.

14 Neves M.M., Marques Jr. A.P., Santana C.V., Lima F.P.C. \& Zambrano W.J. 2002. Características de ovários de fêmeas zebu (Bos taurus indicus), colhidos em abatedouros. Arquivo Brasileiro de Medicina Veterinária e Zootecnia. 54: 1-5. [Fonte: $<$ http://www.scielo.com.br>].

15 Okuda K., Kito S., Sumi N. \& Sato K. 1988. A Study of the central cavity in the bovine corpus luteum. The Veterinary Record. 123: $180-183$.

16 Pathiraja N., Oyedipe E.O. \& Voh A.A. 1986. Accuracy of rectal palpation in the diagnosis of corpora lutea in zebu cows. Brazilian Veterinary Journal. 142: 467-471.

17 Radostitis O.M. \& Blood D.C. 1986. Manual de Controle da Saúde e Produção de Animais. 2.ed. São Paulo: Manole, 189p.

18 Ribadu A.Y., Ward W.R. \& Dobson H. 1994. Comparative evaluation of ovarian structures in cattle by palpation per rectum, ultrasonography and plasma progesterone concentration. Veterinary Review. 135: 452-457.

19 Sisson S. \& Grossman J.D. 1981. Anatomia dos animais domésticos. 5.ed. Rio de Janeiro: Interamericana, 1134p.

20 Soto H.C., González B.H., Rossi M., Godoy S. \& Bello A. 1999. Evaluation de la actividad ovárica de bovinos explotados en condiciones tropicales. Zootecnia Tropical. 17: 3-17.

21 Sprecher D.J., Nibel R.L. \& Whitman S.S. 1989. The predictive value, sensivity and specificity of palpation per rectum and transrectal ultrasonography for the determination of bovine luteal status. Theriogenology. 31: 1165-1172. 